\title{
Nasal Carriage of 200 Patients with Nasal Bone Fracture in Korea
}

\author{
Jun Wook Lee ${ }^{1}$, Young Joon $\mathrm{Kim}^{1}$, Hoon $\mathrm{Kim}^{1}$, Sang Hyun Nam ${ }^{1}$, Bo Moon Shin ${ }^{2}$, \\ Young Woong Choi ${ }^{1}$ \\ Departments of ${ }^{1}$ Plastic and Reconstructive Surgery and ${ }^{2}$ Laboratory Medicine, Sanggye Paik Hospital, Inje University College of Medicine, \\ Seoul, Korea
}

Background Pathogens in the nasal cavity during nasal surgery could lead to a systemic infectious condition, such as bacteremia, nosocomial infection, or toxic shock syndrome. However, there is no research about the prevalence of nasal carriage in patients with nasal bone fracture.

Methods This was a prospective, double-blind, randomized study about the rate of nasal carriage in 200 patients with nasal bone fracture in Korea. Nasal secretions were taken from both the middle nasal meatus and colonized. All analyses were carried out using SPSS software. Results Pathogens were identified in 178 of the 200 cases. Coagulase-negative staphylococci (CNS) were the most cultured bacteria in $127(66.84 \%)$ of the 190 total patients after excluding 10 cases of contaminated samples, and methicillin-resistant coagulase-negative staphylococci (MRCNS) were found in $48(25.26 \%)$. Staphylococcus aureus was the second most identified pathogen, found in 36 (18.95\%), followed by 7 cases (3.68\%) of methicillin-resistant Staphylococcus aureus (MRSA). The prevalence rate of MRSA in the females was higher than that in the males ( $\mathrm{RR}=4.70 ; 95 \% \mathrm{Cl}, 1.09-20.18)$, but other demographic factors had no effect on the prevalence rate of MRSA and MRCNS.

Conclusions The prevalence rate of these pathogens in patients with nasal bone fracture in Korea was similar to other reports. However, few studies have addressed the prevalence rate of CNS and MRCNS in accordance with risk factors or the change in prevalence according to specific prophylaxis against infectious complications. Additional research is needed on the potential connections between clinical factors and microbiological data.

Keywords Nasal bone / Staphylococcus aureus / Methicillin-resistant Staphylococcus aureus
Correspondence: Young Woong Choi Department of Plastic and

Reconstructive Surgery, Sanggye Paik Hospital, Inje University College of

Medicine, 1342 Dongil-ro, Nowon-gu, Seoul 139-707, Korea

Tel: +82-2-950-1048

Fax: +82-2-932-6373

E-mail:pshero2@naver.com

No potential conflict of interest relevant to this article was reported.

Received: 25 Apr 2013 • Revised: 17 Jun 2013 • Accepted: 27 Jun 2013

pISSN: 2234-6163 • elSSN: 2234-6171 • http://dx.doi.org/10.5999/aps.2013.40.5.536• Arch Plast Surg 2013;40:536-541

\section{INTRODUCTION}

Nasal carriage in the healthy population does not raise a clinical problem. During nasal surgery, this normal nasal flora could cross the mucosal barrier and lead to systemic infectious conditions, such as bacteremia, nosocomial infection [1], or toxic shock syndrome (TSS). Staphylococcus aureus (S. aureus) is known to be the most common pathogen in the nasal cavity through many laboratory studies [2,3], and the cause of TSS [4]. Much research has been published on the effect of nasal carriage in special situations, for example the infection rate in patients with chronic sinusitis [5] and the nosocomial infection 
rate [6], the correlation of the prevalence rate of S. aureus with epistaxis [7], and the correlation of the prevalence rate of bacteremia with bleeding during septoplasty [8]. Plastic surgeons have also been concerned about infectious complications by S. aureus during nasal surgery. TSS is a multisystemic disease originating from toxic-shock-syndrome toxin 1 (TSST-1) of $S$. aureus. The incidence rate of TSS after nasal surgery is estimated to be $16.5 / 100,000$, with a mortality rate of approximately $10 \%$ [4]. Nasal bone fracture is the most common trauma to the facial bones, and the nose is a reservoir site of nasal carriage. In nasal bone fracture, the nasal flora can be transmitted to the soft tissue and bloodstream through the external wound directly. When bleeding occurs on reduction of the fractured nasal bone, nasal packing can play a role, much as a tampon used by menstruating women does in cases of TSS [4]. For these reasons, $S$. aureus can lead to fatal complications after nasal bone fracture.

Coagulase-negative staphylococci (CNS) had been regarded as apathogenic normal flora and disseminated culture contamination for a long time. Then Huebner and Goldmann [9] insisted that CNS play a role as pathogens. Furthermore, the increase in the prevalence rate of methicillin-resistant coagulase-negative staphylococci (MRCNS) has been noteworthy. MRCNS is disseminated in the community and may act as a reservoir for staphylococcal cassette chromosome mec (SCCmec) to convert from S. aureus to methicillin-resistant S. aureus (MRSA) [10]. Although the specific virulence factor has not been determined, it is clear that a CNS infection can lead to infectious complications (e.g., catheter-related infection, meningitis, endocarditis, urinary tract infection) [9] and MRCNS is a pool of SCCmec. However, no research on the prevalence of nasal carriage of CNS and MRCNS in healthy populations and patients under the specific conditions. Therefore, this prospective, double-blind, randomized study investigated the infection rate of nasal carriage in 200 patients with nasal bone fracture in Korea.

\section{METHODS}

A prospective study was conducted on 200 patients at our department in Korea from July 1, 2010 to March 31, 2011. There were no exclusion criteria except cases in which patients could not be operated on under general anesthesia. Patients hospitalized through the emergency center or outpatient department were diagnosed with nasal bone fracture by 3-dimensional facial computed tomography. All of the patients who were classified by severity by Rohrich and Adams [11] underwent closed reduction under general anesthesia regardless of the criteria and gave consent before being accepted into the study. None of the patients were injected with intravenous antibiotics. Preopera- tive samples were taken by the same method. Postoperatively, the study groups were divided to four groups through intranasal irrigation with (or without) antibiotics (first generation cephalosporin) and nasal packing with (or without) mupirocin ointment. In this article, the preoperative samples were analyzed.

To prevent contamination, 400 samples were taken intraoperatively after surgical draping in the operating room. Each nasal secretion was taken from both middle nasal meatus and put on Stuart agar gel medium with a sterile transport swab (Transystem, Murrieta, CA, USA). On collecting the samples, the nasal secretions were taken through the nasal speculum to avoid contamination from the nasal vestibule. The sample immediately transported to the microbiological laboratory was cultured and identified by the Department of Laboratory Medicine. Classification of the bacteriological findings was made in four main categories: a group of colonized bacteria that were potential bacterial pathogens, a group that grew non-pathogenic normal flora, a group that grew no bacteria, and a group that was contaminated. A sample was counted as the group that grew pathogens if one of the two samples contained colonized pathogens or normal flora. In the 'no culture' group, no organisms grew in either nasal cavity, and in the contaminated groups, contamination was found in samples from both cavities. CNS were considered pathogens, and thus included in the 'pathogen growth' group in this study, like other recent studies $[4,9]$.

All of the analyses were carried out using PASW ver. 18.0 (SPSS Inc., Chicago, IL, USA). The results are expressed as mean \pm standard deviation unless otherwise specified. The unpaired Student's t-test was used to analyze continuous data, and either the 2 -tailed chi-squared test ( $\chi^{2}$ test) or Fisher's exact test was used to analyze categorical data, where appropriate, with 2-tailed relative risk (RR). The epistaxis, severity, and hospitalization were used to compare between groups. Results with $\mathrm{P}<0.05$ were considered statistically significant.

\section{RESULTS}

Pathogens were identified in the nasal cavities of 178 of the 200 patients (Table 1). 10 samples were contaminated, and those were excluded from the total number of patients. No colonized groups were identified in 12 , but these groups were included in total numbers. There was no normal flora containing aerobic strains such as S. pyogenes or pneumonia [2]. CNS were evaluated as a pathogen in this study [9]. CNS were the most cultured bacteria, found in 127 (66.84\%) of the 190 total patients after the 10 contaminated cases had been excluded, and MRCNS was found in 48 (25.26\%). S. aureus was the second most identified pathogen in 36 (18.95\%), followed by MRSA in 
7 (3.68\%). In 14 subjects (7.37\%), various Gram-negative rods were identified, among them Enterobacter aerogenes, Raoultella planticola, the Proteus vulgaris group, Haemophilus influenza, Serratia marcescens, and Streptococcus mitis. Fungus was found in 1 sample and anaerobes were not found in the study. In other studies, the CNS was the most colonized pathogen. In a study by Gluck and Gebbers [2], CNS was regarded as normal flora, not a pathogenic bacterium, and excluded from the list of nasal flora. Because S. epidermidis is a type of CNS $[9,10]$, CNS were also the most common nasal flora in a prevalence report by Savolainen et al. [12] (Table 2).

Table 1. Microbiologic results from fractioned swabs of the nasal cavity of 200 patients with nasal bone fracture

\begin{tabular}{|lc|}
\hline Bacteria & Patients in positive culture \\
\hline Growth & $178(93.68)$ \\
Aerobic bacteria & $177(93.16)$ \\
Gram-positive bacteria & $163(85.79)$ \\
Coagulase negative staphylococci & $127(66.84)$ \\
MRCNS & $48(25.26)$ \\
Staphylococcus aureus & $36(18.95)$ \\
MRSA & $7(3.68)$ \\
Gram-negative bacteria & $14(7.37)$ \\
Enterobacter aerogenes & $8(4.21)$ \\
Raoultella planticola & $1(0.53)$ \\
Proteus vulgaris group & $1(0.53)$ \\
Haemophilus influenzae & $1(0.53)$ \\
Klebsiella pneumoniae & $1(0.53)$ \\
Serratia marcescens & $1(0.53)$ \\
Streptococcus mitis & $1(0.53)$ \\
Anaerobic bacteria & $0(0)$ \\
Fungus & $1(0.53)$ \\
Cryptococcus species & $1(0.53)$ \\
No growth & $12(6.32)$ \\
Total & $190(100)$ \\
\hline Values are presented as number (\%). & \\
MRCNS, methicillin-resistant coagulase-negative staphylococci; MRSA, methicillin- \\
resistant Staphylococcus aureus. \\
\hline
\end{tabular}

The demographic and clinical characteristics of patients with and without S. aureus, MRSA, CNS, and MRCNS colonization are shown in Tables 3, 4. The mean and standard deviation of the patient age and duration (day) from trauma to culture were calculated, and a comparison of prevalence by sex was measured by relative risk. S. aureus was identified in 36 (18.95\%) of the 190 samples. The mean age of the patients with S. aureus was 4.45 less than that of the patients without $S$. aureus $(\mathrm{P}=$ 0.0735). The prevalence rate of S. aureus in females was $3.17 \%$ more than that in males ( $\mathrm{RR}=1.17 ; 95 \% \mathrm{CI}, 0.60-2.30)$. Of the 190 cultures obtained, 7 (3.68\%) were positive for MRSA by the culture method, and 29 (15.26\%) were susceptible to methicillin. MRSA accounted for 7 (19.44\%) of 36 S. aureus isolates. By univariate analysis, colonization of MRSA in the females was $14.28 \%$ higher than that in the males $(\mathrm{RR}=4.70$; $95 \% \mathrm{CI}, 1.09$ 20.18), and the prevalence rate of MRSA was $7.49 \%$ more in the females than in the males $(\mathrm{P}=0.023)$. MRSA was more prevalent in the severely fractured group and epistaxis group (1.36\% and $5.11 \%)$, but this difference was not statistically significant $(\mathrm{P}=0.685, \mathrm{P}=0.094)$. Moreover, MRSA was less prevalent in those hospitalized for a longer duration (Table 5).

CNS was colonized in 127 (66.84\%) and MRCNS in 48 (25.26\%) of 190 cases without contaminated samples. MRCNS accounted for 48 (37.80\%) of 127 CNS isolates. MRCNS was more prevalent in the cases with epistaxis, the mildly fractured, and younger age groups, but these findings were not statistically significant (Table 6).

\section{DISCUSSION}

Recent concerns about increases in the incidence of MRSA infections in the community have lent surveillance of $S$. aureus greater importance. Previous research traced an association between $S$. aureus nasal carriage and subsequent infection among

Table 2. Comparison of the nasal flora in the healthy populations from other studies with Korean patients presenting with nasal bone fracture

\begin{tabular}{|c|c|c|c|c|c|c|c|c|}
\hline \multirow{10}{*}{$\begin{array}{l}\text { Colonized bacteria } \\
\text { in nasal cavity }\end{array}$} & \multicolumn{2}{|c|}{$\begin{array}{c}\text { By Savolainen et al. [12], } \\
1986\end{array}$} & \multicolumn{2}{|c|}{$\begin{array}{l}\text { By Gluck and Gebbers [2], } \\
2000\end{array}$} & \multicolumn{2}{|c|}{$\begin{array}{c}\text { By Kirtsreesakul et al. [3], } \\
2008\end{array}$} & \multicolumn{2}{|c|}{ By our group } \\
\hline & S. epidermidis & 79 & S. aureus (MRSA) & $41(0.97)$ & CNS & 61.6 & CNS (MRCNS) & $66.84(25.26)$ \\
\hline & Diphtheroids & 41 & Citrobacter diversus & 12 & S. aureus & 11.6 & S. aureus (MRSA) & $18.95(3.68)$ \\
\hline & S. aureus & 34 & Haemophilus parainfluenzae & 11 & Corynebacterium & 9.3 & Enterobacter aerogenes & 4.21 \\
\hline & Haemophilus influenzae & 5 & Haemophilus influenzae & 10 & Enterobacter aerogenes & 4.7 & Raoultella planticola & 0.53 \\
\hline & a-Hemolytic streptococci & 4 & Moraxella catarrhalis & 8 & Citrobacter diversus & 3.5 & Proteus vulgaris group & 0.53 \\
\hline & & & & & Klebsiella pneumonia & 3.5 & Haemophilus influenzae & 0.53 \\
\hline & & & & & & & Klebsiella pneumoniae & 0.53 \\
\hline & & & & & & & Serratia marcescens & 0.53 \\
\hline & & & & & & & Streptococcus mitis & 0.53 \\
\hline
\end{tabular}


Table 3. Demographic characteristics of patients with nasal bone fracture tested for nasal colonization

\begin{tabular}{|c|c|c|c|c|c|c|c|c|c|}
\hline \multirow[b]{2}{*}{ Characteristic } & \multirow[b]{2}{*}{ All patients } & \multicolumn{4}{|c|}{ Staphylococcus aureus } & \multicolumn{4}{|c|}{ MRSA } \\
\hline & & Yes $(n=36)$ & No $(n=164)$ & RR $(95 \% \mathrm{Cl})$ & $\begin{array}{c}\mathrm{P}- \\
\text { value }^{\text {a) }}\end{array}$ & Yes $(n=7)$ & No $(n=193)$ & RR $(95 \% \mathrm{Cl})$ & $\begin{array}{c}\mathrm{P}- \\
\text { value }^{\text {a) }}\end{array}$ \\
\hline Age (yr) & $29.85 \pm 16.70$ & $26.2 \pm 15.62$ & $30.65 \pm 16.86$ & $(-10.50-1.59)$ & 0.07 & $23.14 \pm 15.16$ & $30.09 \pm 16.73$ & $(-19.61-5.71)$ & 0.14 \\
\hline \multicolumn{10}{|l|}{ Sex } \\
\hline Female & 42 & 9 (21.42) & 33 & $1.17(0.60-2.30)$ & & $4(9.52)$ & 38 & $4.70(1.09-20.18)$ & \\
\hline Male & 148 & 27 (18.24) & 121 & & & $3(2.02)$ & 145 & & \\
\hline $\begin{array}{l}\text { Days from trauma } \\
\text { to culture }\end{array}$ & $5.91 \pm 2.82$ & $6.36 \pm 3.55$ & $5.81 \pm 2.63$ & $(-0.48-1.57)$ & 0.15 & $5.49 \pm 2.70$ & $5.93 \pm 2.83$ & $(-2.65-1.64)$ & 0.32 \\
\hline
\end{tabular}

Table 4. Demographic characteristics of patients with nasal bone fracture tested for nasal colonization

\begin{tabular}{|c|c|c|c|c|c|c|c|c|c|}
\hline \multirow[b]{2}{*}{ Characteristics } & \multirow[b]{2}{*}{ All patients } & \multicolumn{4}{|c|}{ CNS } & \multicolumn{4}{|c|}{ MRCNS } \\
\hline & & Yes $(n=127)$ & No $(n=73)$ & $\mathrm{RR}(95 \% \mathrm{Cl})$ & $\begin{array}{c}\mathrm{P}- \\
\text { value }^{\mathrm{a})}\end{array}$ & Yes $(n=48)$ & No $(n=152)$ & $\mathrm{RR}(95 \% \mathrm{Cl})$ & $\begin{array}{c}\mathrm{P}- \\
\text { value }^{\mathrm{a})}\end{array}$ \\
\hline Age (yr) & $29.85 \pm 16.70$ & $30.47 \pm 16.57$ & $28.77 \pm 16.97$ & $(-3.14-6.55)$ & 0.24 & $29.54 \pm 16.51$ & $29.94 \pm 16.80$ & $(-5.87-5.06)$ & 0.44 \\
\hline \multicolumn{10}{|c|}{ 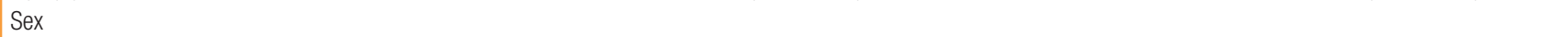 } \\
\hline Female & 42 & 28 (66.67) & 14 & $0.990(0.78-1.26)$ & & $10(23.81)$ & 32 & $0.927(0.51-1.70)$ & \\
\hline Male & 148 & 99 (66.89) & 148 & & & 38 (25.68) & 110 & & \\
\hline $\begin{array}{l}\text { Days from trauma to } \\
\text { culture }\end{array}$ & $5.91 \pm 2.82$ & $5.75 \pm 2.74$ & $6.21 \pm 2.95$ & $(-1.29-0.36)$ & & $6.09 \pm 3.08$ & $5.86 \pm 2.75$ & $(-0.71-1.15)$ & 0.32 \\
\hline
\end{tabular}

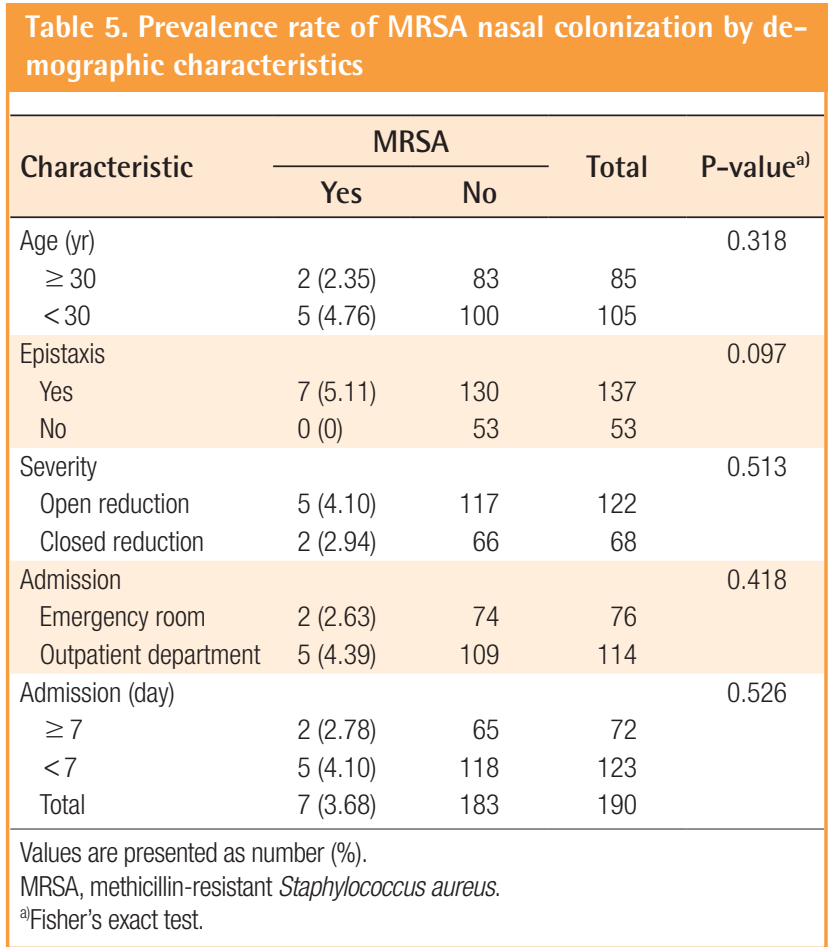

patients in both health care and community settings [13]. The use of antibiotics may eliminate methicillin-sensitive $S$. aureus and allow for the proliferation of MRSA [14]. A study by Kuehnert et al. [15] on the incidence of S. aureus and MRSA in a US population reported rates of approximately $32.4 \%$ and $0.8 \%$, respectively. The prevalence rate of this bacteria may vary with demographic patterns such as age, sex, and race. The incidence of $S$. aureus and MRSA in the Swiss was found to be $41 \%$ and $0.97 \%$, respectively [2]. On the other hand, the incidence of $S$. aureus and MRSA in this study was $18.98 \%$ and $3.68 \%$, respectively. The present study found that MRSA colonization was associated with females, as in the US study. The prevalence of $S$. aureus and MRSA was found to be $33 \%$ and $2.2 \%$, respectively, in healthy Koreans according to Kim et al. [16]. Sampling from healthy populations was cultured and performed with an aseptic maneuver in previous studies on nasal carriage, although the exact method may have varied from study to study, thus causing variation in results. However, the reason for the prevalence pattern was not known and the small sample size in this study prevented making a statistically significant comparison with the data from Kuehnert et al. [15]. Furthermore, the prevalence rate of MRSA in this study was higher than that in previous studies such as Gluck and Gebbers [2] and Kuehnert et al. [15], and was similar to that in the study by Kim et al. [16]. As regional 
Table 6. Comparisons of the prevalence rate of MRCNS nasal colonization by demographic characteristics

\begin{tabular}{|c|c|c|c|c|c|}
\hline \multirow[b]{2}{*}{ Characteristic } & \multicolumn{2}{|c|}{ MRCNS } & \multirow[b]{2}{*}{ Total } & \multirow{2}{*}{$\begin{array}{l}\text { Chi- } \\
\text { square } \\
\text { test } \\
(\mathrm{df}=1)\end{array}$} & \multirow{2}{*}{$\begin{array}{c}\mathrm{P}- \\
\text { value }^{\mathrm{a}}\end{array}$} \\
\hline & Yes & No & & & \\
\hline Sex & & & & 0.060 & 0.806 \\
\hline Female & $10(23.81)$ & 32 & 42 & & \\
\hline Male & 38 (25.68) & 110 & 148 & & \\
\hline Age (yr) & & & & 0.690 & 0.406 \\
\hline$\geq 30$ & 19 (22.35) & 66 & 85 & & \\
\hline$<30$ & 29 (27.61) & 76 & 105 & & \\
\hline Epistaxis & & & & 0.268 & 0.605 \\
\hline Yes & $36(26.28)$ & 101 & 137 & & \\
\hline No & $12(22.64)$ & 41 & 53 & & \\
\hline Severity & & & & 3.146 & 0.076 \\
\hline Open reduction & 26 (21.31) & 96 & 122 & & \\
\hline Closed reduction & $22(32.35)$ & 46 & 68 & & \\
\hline Admission & & & & 0.562 & 0.453 \\
\hline Emergency room & 17 (22.37) & 59 & 76 & & \\
\hline Outpatients department & $31(27.20)$ & 83 & 114 & & \\
\hline Admission (day) & & & & 0.525 & 0.469 \\
\hline$\geq 7$ & 19 (28.36) & 48 & 67 & & \\
\hline$<7$ & $29(23.58)$ & 94 & 123 & & \\
\hline Total & $48(25.26)$ & 142 & 190 & & \\
\hline \multicolumn{6}{|c|}{$\begin{array}{l}\text { Values are presented as number (\%). } \\
\text { MRCNS, methicillin-resistant coagulase-negative staphylococci; OR, open reduction; } \\
\text { CR, closed reduction; ER, emergency room, OPD; outpatients department. } \\
\text { a)Chi-square test. }\end{array}$} \\
\hline
\end{tabular}

differences in the prevalence of MRSA have not been identified, analyzing the prevalence according to genetic factors (e.g., ethnicity), and social factors (e.g., frequency of antibiotic use).

CNS was the most common nasal infection (66.84\%), and the incidence of MRCNS was $25.26 \%$. CNS was considered as normal nasal flora in most studies about nasal carriage. According to the latest edition of the American Society of Microbiology's Manual of Clinical Microbiology [17], the normal nasal flora contains aerobic strains such as S. pyogenes and pneumoniae, CNS, corynebacteria, stomatococcus, micrococcus, lactobacilli, and mycoplasmas. However, CNS has been regarded as a pathogen leading to infectious complications in recent studies [9], and MRCNS has been regarded as a reservoir of SCCmec that converts $S$. aureus to MRSA [18]. Although the recent increase in MRCNS colonization and the toxicities of MRCNS are clear, studies on the incidence of MRCNS and related factors have been inadequate. MRCNS were probably disseminated in the community, notably in subjects without previous exposure to the health care system [10]. More laboratory and clinical studies about MRCNS are necessary to reduce infection-related MRSA and MRCNS.

Infectious complications of nasal surgery vary from local (septal hematoma, abscess, and cellulitis) to systemic (toxic shock syndrome) [19]. Nasal carriage of pathogens such as S. aureus and MRSA could cause systemic infection. S. aureus bacteremia appears to be of endogenous origin since the bacteria originate from colonies in the nasal mucosa [13]. Actually bacteremia was more frequently encountered among those with a greater amount of bleeding during the nasal surgery by Koc et al. [8]. Patients with bleeding have an increased risk of developing bacteremia that could produce systemic complications, and Perl [20] described advances in the prevention of S. aureus infections from endogenous colonization in 2003. It was reported that $S$. aureus carriers have a 2- to 9-fold greater rate of development of surgical site or intravenous catheter infection. It is also important to consider measures to prevent surgical infections in nasal surgery patients because of the difficulties in treatment, although the incidence of MRSA infection is low $[21,22]$. To reduce and prevent against infection of these bacteria, various strategies have been introduced.

Three strategies [21] for eliminating nasal carriage have been identified: locally applied antibiotics or disinfectants, systemic antibiotics, and bacterial interference. Of the commonly used agents, mupirocin (pseudomonic acid) ointment has been shown to be $97 \%$ effective in reducing S. aureus nasal carriage. Infection rates were also decreased, but the infection rate in surgical sites was not reduced significantly compared with a placebo group [22]. Furthermore, locally applied and systemic antibiotics have variable efficacy and frequently cause antimicrobial resistance. Routine antibiotic prophylaxis was found to be redundant because the infection rate after elective nasal surgery (e.g. corrective rhinoplasty) was very low according to the review of Koc et al. [8]. However, patients with nasal bone fracture who had external wounds on their nose had an increased risk of infection compared with corrective rhinoplasty. Therefore, antibiotic prophylaxis is necessary to prevent infectious complications. In reports by Rajan et al. [23] and Gocer et al. [24], systemic antibiotic prophylaxis was found to be effective in controlling the rate of nasal carriage. Furthermore, a single-dose injection of antibiotics is a more attractive regimen than combined antibiotic injection. The effectiveness is similar in both therapies and the cost is lower in single dose injection. However, few studies have addressed the change in prevalence of nasal carriage according to specific prophylaxis against infectious complications.

In conclusion, the prevalence rate of pathogens such as S. aureus, MRSA, CNS, and MRCNS in patients with nasal bone fracture in Korea was similar to those of other reports. It remains necessary to investigate the correlation between microbiological test data and many clinical factors such as infectious complications and management. 


\section{REFERENCES}

1. van Rijen MM, Bonten M, Wenzel RP, et al. Intranasal mupirocin for reduction of Staphylococcus aureus infections in surgical patients with nasal carriage: a systematic review. J Antimicrob Chemother 2008;61:254-61.

2. Gluck U, Gebbers JO. The nose as bacterial reservoir: important differences between the vestibule and cavity. Laryngoscope 2000; 110:426-8.

3. Kirtsreesakul V, Tuntaraworasin J, Thamjarungwong B. Microbiology and antimicrobial susceptibility patterns of commensal flora in the middle nasal meatus. Ann Otol Rhinol Laryngol 2008; 117:914-8.

4. Breda SD, Jacobs JB, Lebowitz AS, et al. Toxic shock syndrome in nasal surgery: a physiochemical and microbiologic evaluation of Merocel and NuGauze nasal packing. Laryngoscope 1987;97:1388-91.

5. Kremer B, Jacobs JA, Soudijn ER, et al. Clinical value of bacteriological examinations of nasal and paranasal mucosa in patients with chronic sinusitis. Eur Arch Otorhinolaryngol 2001;258:220-5.

6. Perl TM, Golub JE. New approaches to reduce Staphylococcus aureus nosocomial infection rates: treating $S$. aureus nasal carriage. Ann Pharmacother 1998;32:S7-16.

7. Whymark AD, Crampsey DP, Fraser L, et al. Childhood epistaxis and nasal colonization with Staphylococcus aureus. Otolaryngol Head Neck Surg 2008; 138:307-10.

8. Koc S, Uysal IO, Uysal EB, et al. The comparison of bacteremia and amount of bleeding during septoplasty. Eur Arch Otorhinolaryngol 2012;269:1139-42.

9. Huebner J, Goldmann DA. Coagulase-negative staphylococci: role as pathogens. Annu Rev Med 1999;50:223-36.

10. Barbier F, Ruppe E, Hernandez D, et al. Methicillin-resistant coagulase-negative staphylococci in the community: high homology of SCCmec IVa between Staphylococcus epidermidis and major clones of methicillin-resistant Staphylococcus aureus. J Infect Dis 2010;202:270-81.

11. Rohrich RJ, Adams WP Jr. Nasal fracture management: minimizing secondary nasal deformities. Plast Reconstr Surg 2000;106:266-73.

12. Savolainen S, Ylikoski J, Jousimies-Somer H. The bacterial flora of the nasal cavity in healthy young men. Rhinology $1986 ; 24: 249-55$.
13. von Eiff C, Becker K, Machka K, et al. Nasal carriage as a source of Staphylococcus aureus bacteremia. Study Group. N Engl J Med 2001;344:11-6.

14. Kluytmans J, van Belkum A, Verbrugh H. Nasal carriage of Staphylococcus aureus: epidemiology, underlying mechanisms, and associated risks. Clin Microbiol Rev 1997;10: 505-20.

15. Kuehnert MJ, Kruszon-Moran D, Hill HA, et al. Prevalence of Staphylococcus aureus nasal colonization in the United States, 2001-2002. J Infect Dis 2006;193:172-9.

16. Kim HB, Shin DH, Park KU, et al. The methicillin-resistance rate of Staphylococcus aureus isolatd from anterior nares of healthy adults in the community. Korean J Infect Dis 1998; 30:527-31.

17. Versalovic J, American Society for Microbiology. Manual of clinical microbiology. 10th ed. Washington, DC: American Society for Microbiology; 2011.

18. Otto M. Coagulase-negative staphylococci as reservoirs of genes facilitating MRSA infection: Staphylococcal commensal species such as Staphylococcus epidermidis are being recognized as important sources of genes promoting MRSA colonization and virulence. Bioessays 2013;35:4-11.

19. Cochran CS, Landecker A. Prevention and management of rhinoplasty complications. Plast Reconstr Surg 2008;122: 60e-7e.

20. Perl TM. Prevention of Staphylococcus aureus infections among surgical patients: beyond traditional perioperative prophylaxis. Surgery 2003;134:S10-7.

21. Angelos PC, Wang TD. Methicillin-resistant Staphylococcus aureus infection in septorhinoplasty. Laryngoscope 2010; 120:1309-11.

22. van Rijen M, Bonten M, Wenzel R, et al. Mupirocin ointment for preventing Staphylococcus aureus infections in nasal carriers. Cochrane Database Syst Rev 2008;(4): CD006216.

23. Rajan GP, Fergie N, Fischer U, et al. Antibiotic prophylaxis in septorhinoplasty? A prospective, randomized study. Plast Reconstr Surg 2005;116:1995-8.

24. Gocer C, Dursun E, Acar A, et al. Antimicrobial prophylaxis in nasoseptal surgery [Internet]. Ankara: KBB-Forum; 2007 [cited 2013 Aug 28]. Available from: http://www.kbbforum. net/journal/pdf/pdf_KBB_170.pdf. 